\title{
Fixed Point Theorems for Cyclic Weakly Contraction Mappings in Dislocated Quasi Extended $b$-Metric Space
}

\author{
Budi Nurwahyu \\ Department of Mathematics, Hasanuddin University, Tamalanrea KM 10, Makassar, Indonesia \\ Correspondence should be addressed to Budi Nurwahyu; budinurwahyu@unhas.ac.id
}

Received 21 May 2019; Accepted 24 July 2019; Published 6 August 2019

Academic Editor: Vakhtang M. Kokilashvili

Copyright (c) 2019 Budi Nurwahyu. This is an open access article distributed under the Creative Commons Attribution License, which permits unrestricted use, distribution, and reproduction in any medium, provided the original work is properly cited.

In this paper, we establish and prove some theorems about existence and uniqueness of fixed point for cyclic weakly contraction mappings in dislocated quasi extended $b$-metric space.

\section{Introduction}

One of the famous generalizations of metric space which was introduced by Bakhtin in 1989 [1] is $b$-metric space. Many authors utilized the space for fixed point results on contraction mapping or weakly contraction mapping, such as Saluja et al. [2], Mostefaoui et al. [3], Chaudhury et al. [4] and Ansari et al. [5]. In 2012, Shah et al. [6] introduced quasi $b$-metric space which removed symmetric conditions in $b$-metric and for utilizing in common fixed point results on contraction mapping. Some authors such as Zhu et al. [7] and Cvetkovic et al. [8] gave some results in that space. In 2013, Hussain et al. [9] introduced dislocated $b$-metric which weakened first condition in $b$-metric for fixed point results, and Rasham et al. [10] utilized the space for multivalued fixed point results. In 2016, Rahman et al. [11] generalized the dislocated $b$-metric to be dislocated quasi $b$-metric. Several papers has published in dislocated quasi $b$-metric for containing fixed point results on generalized Banach contraction mappings, such as Klin-eam et al. [12], Suanom et al. [13], and Tiwari et al. [14]. Recently, in 2017, Kamran et al. [15] generalized triangular inequality condition on $b$-metric such that to be extended $b$-metric and utilized the space for fixed point results. Samreen et al. [16] yielded some theorems for fixed point results on nonlinear contraction mappings in the space and Alqahtani et al. [17, 18] utilized the space for common fixed point results on two self-mappings and on Kcontraction mapping.

Inspired by the extended $b$-metric space of Samreen et al. [16]. In this work, we introduced a concept of dislocated quasi extended $b$-metric space as a generalization of dislocated quasi $b$-metric space [11]. We establish and prove some fixed point theorems in the dislocated quasi extended $b$-metric space, by utilizing weakly contraction mapping which was introduced by Rhoades [19] and cyclic contraction which was introduced by Zoto et al. [20]. In addition, we also provide some examples to clarify the theorems.

\section{Preliminaries}

In the following section, we need some definitions to govern and prove our theorems.

Definition 1 (see [1]). Let $X$ be a non-empty set and a real number $k \geq 1$. Let $d: X \times X \longrightarrow[0, \infty)$ be a function. The pair $(X, d)$ is called $b$-metric space if the following conditions are satisfied:

(1) $d(x, y)=0$ if and only if $x=y$,

(2) $d(x, y)=d(y, x)$,

(3) $d(x, y) \leq k(d(x, z)+d(z, y))$,

for all $x, y, z \in X$.

Example 2 (see [15]). Let $X=l_{p}(R)$ with $0<p<1$, where $l_{p}(R)=\left\{\left\{a_{k}\right\} \subseteq R \mid \sum_{k=1}^{\infty} a_{k}<\infty\right\}$. Let $d: X \times X \longrightarrow$ $[0, \infty)$ be a function, which is defined as $d(x, y)=\sum_{k=1}^{\infty} \mid a_{k}-$ $\left.b_{k}\right|^{1 / p}$, where $x=\left\{a_{k}\right\}$ and $y=\left\{b_{k}\right\}$. Then $d$ is a $b$-metric with parameter $b=2^{1 / p}$. 
Definition 3 (see [11]). Let $X$ be a nonempty set and a real number $k \geq 1$. Let $d: X \times X \longrightarrow[0, \infty)$ be a function. The pair $(X, d)$ is called a dislocated quasi b-metric space (in short $d q b$ - metric space) if the following conditions are satisfied:

(1) $d(x, y)=0$ then $x=y$,

(2) $d d(x, y) \leq k(d(x, z)+d(z, y))$,

for all $x, y, z \in X$.

Example 4 (see [11]). Let $X=R$ and define $d(x, y)=\mid 2 x-$ $\left.y\right|^{2}+|2 x+y|^{2}$. It is easy to show that $(X, d)$ is a dislocated quasi $b$-metric space with $k=2$.

Definition 5 (see [15]). Let $X$ be a non-empty set and $k: X \times$ $X \longrightarrow[1, \infty)$ be a function. Let $d: X \times X \longrightarrow[0, \infty)$ be a function. The pair $(X, d)$ is called an extended $b$-metric space if the following conditions are satisfied:

(1) $d(x, y)=0$ if and only if $x=y$,

(2) $d(x, y)=d(y, x)$,

(3) $d(x, y) \leq k(x, y)(d(x, z)+d(z, y))$,

for all $x, y, z \in X$.

Example 6 (see [16]). Let $X=\{1,2,3, \ldots\}$. Define $k: X \times$ $X \longrightarrow[1, \infty)$ and $d: X \times X \longrightarrow[0, \infty)$ as follows:

$$
k(x, y)= \begin{cases}|x-y|^{2} & \text { if } x \neq y \\ 1 & \text { if } x=y\end{cases}
$$

$$
\text { and } d(x, y)=(x-y)^{4} \text {. }
$$

It is easy to show that $(X, d)$ is a dislocated extended $b$-metric space.

Definition 7. Let $X$ be a non-empty set and $k: X \times X \longrightarrow$ $[1, \infty)$. Let $d_{k}: X \times X \longrightarrow[0, \infty)$ be a function. The pair $\left(X, d_{k}\right)$ is called a quasi extended b-metric space (in short qebmetric space) if the following conditions are satisfied:

$$
\begin{aligned}
& \text { (1) } d_{k}(x, y)=0 \text { if and only if } x=y \text {, } \\
& \text { (2) } d_{k}(x, y) \leq k(x, y)\left(d_{k}(x, z)+d_{k}(z, y)\right) \text {, }
\end{aligned}
$$

for all $x, y, z \in X$.

Example 8. Let $X=[0,1]$ and $d(x, y)=\left|2^{x-y}-1\right|$ for $x, y \in$ $[0,1]$. Let $k(x, y)=2^{1-(x+y) / 2}$ for $x, y \in[0,1]$.

It is obvious that for first condition and $d(x, y)$ is not symmetric. For second condition, consider that

$$
\begin{aligned}
& 2^{1-(x+y) / 2}(d(x, z)+d(z, y)) \\
& \quad=2^{1-(x+y) / 2}\left(\left|2^{x-z}-1\right|+\left|2^{z-y}-1\right|\right)
\end{aligned}
$$

Since $\min _{z \in[0,1]}\left|2^{x-z}-1\right|+\left|2^{z-y}-1\right|=\left|2^{x-(x+y) / 2}-1\right|+$ $\left|2^{(x+y) / 2-y}-1\right|$, we get

$$
\begin{aligned}
& 2^{1-(x+y) / 2}\left(\left|2^{x-z}-1\right|+\left|2^{z-y}-1\right|\right) \\
& \quad \geq 2^{1-(x+y) / 2}\left(\left|2^{x-(x+y) / 2}-1\right|+\left|2^{(x+y) / 2-y}-1\right|\right) \\
& \quad=2^{1-\lfloor(x+y) / 2\rfloor}\left(\left|2^{(x-y) / 2}-1\right|+\left|2^{(x-y) / 2}-1\right|\right) \\
& \quad=2^{2-(x+y) / 2}\left(\left|2^{(x-y) / 2}-1\right|\right) .
\end{aligned}
$$

If $x \leq y$ then we have $(x+y) / 2 \geq x$, and $2^{2-(x+y) / 2} \leq 2^{2-x}$. Therefore, we get

$$
\begin{aligned}
& 2^{1-(x+y) / 2}\left(\left|2^{x-z}-1\right|+\left|2^{z-y}-1\right|\right) \\
& \quad \geq\left(\left|2^{2-y}-2^{2-(x+y) / 2}\right|\right) \geq\left(\left|2^{2-y}-2^{2-x}\right|\right) \\
& \quad=2^{2}\left(\left|2^{-y}-2^{-x}\right|\right)
\end{aligned}
$$

Since, for $x \in[0,1], 2-x \geq 1$. Thus we get

$$
\begin{aligned}
& 2^{1-(x+y) / 2}\left(\left|2^{x-z}-1\right|+\left|2^{z-y}-1\right|\right. \\
& \quad \geq 2^{2}\left(2^{-x}\right)\left(\left|2^{x-y}-1\right|\right)=2^{2-x}\left|2^{x-y}-1\right| \\
& \quad \geq\left|2^{x-y}-1\right|=d(x, y)
\end{aligned}
$$

If $x \geq y$ then we have $2^{2-y} \geq 2^{2-x},(x+y) / 2 \leq y$, and $2^{2-(x+y) / 2} \leq 2^{2-y}$. Thus we get

$$
\begin{aligned}
& 2^{1-(x+y) / 2}\left(\left|2^{x-z}-1\right|+\left|2^{z-y}-1\right|\right. \\
& \quad \geq\left(\left|2^{2-y}-2^{2-(x+y) / 2}\right|\right) \geq\left(\left|2^{2-x}-2^{2-y}\right|\right) \\
& \quad=2^{2}\left(\left|2^{-x}-2^{-y}\right|\right)=2^{2}\left(\left|2^{-y}-2^{-x}\right|\right) \\
& \quad=2^{2}\left(2^{-x}\right)\left(\left|2^{x-y}-1\right|\right)=2^{2-x}\left|2^{x-y}-1\right| \\
& \quad \geq\left|2^{x-y}-1\right|=d(x, y) .
\end{aligned}
$$

Hence, we have

$$
d(x, y) \leq 2^{1-(x+y) / 2}(d(x, z)+d(z, y)) .
$$

Thus $d$ is a quasi $b$-metric in $X=[0,1]$.

Definition 9. Let $X$ be a non-empty set and $k: X \times X \longrightarrow$ $[1, \infty)$ and let $d_{k}: X \times X \longrightarrow[0, \infty)$ be a function. The pair $\left(X, d_{k}\right)$ is called a dislocated quasi extended $b$-metric space (in short dqeb-metric space) if the following conditions are satisfied:

$$
\begin{aligned}
& \text { (1) } d_{k}(x, y)=0 \text { then } x=y, \\
& \text { (2) } d_{k}(x, y) \leq k(x, y)\left(d_{k}(x, z)+d_{k}(z, y)\right) \text {, }
\end{aligned}
$$

for all $x, y, z \in X$.

Remark 10. If $k(x, y)=k \geq 1$, then $d q e b$ is $d q b$. 
Example 11. Let $X=[-1,1]$ and $d_{k}(x, y)=(|x|+|y|)+$ $|x|^{2} / m+|y|^{2} / n, m \neq n$, for $x, y \in[-1,1]$.

Let $k(x, y)=(2+|x y|) / 2$ for $x \in[-1,1]$.

In fact, it is clear that if $d(x, y)=0$, then $x=y=0$, which is satisfied for first condition. For second condition, we consider,

$$
\begin{aligned}
& k(x, y)\left(d_{k}(x, z)+d_{k}(z, y)\right)=\frac{2+|x y|}{2}((|x|+|z|) \\
& \left.+\frac{|x|^{2}}{m}+\frac{|z|^{2}}{n}+(|z|+|y|)+\frac{|z|^{2}}{m}+\frac{|y|^{2}}{n}\right) \\
& \quad \geq \frac{2+|x y|}{2}\left((|x|+|y|)+\frac{|x|^{2}}{m}+\frac{|z|^{2}}{n}+\frac{|z|^{2}}{m}\right. \\
& \left.+\frac{|y|^{2}}{n}\right) \geq\left((|x|+|y|)+\frac{|x|^{2}}{m}+\frac{|y|^{2}}{n}\right) \\
& \quad=d_{k}(x, y) .
\end{aligned}
$$

Thus $\left(X, d_{k}\right)$ is a dislocated quasi extended $b$-metric space, with $k(x, y)=(2+|x y|) / 2 \geq 1$.

Definition 12 (see $[12,13])$. Let $\left(X, d_{k}\right)$ be a dislocated quasi extended $b$-metric space and let $\left\{x_{n}\right\}$ be a sequence in $X$.

(i) $\left\{x_{n}\right\}$ convergent sequence to $x \in X$, if $\lim _{n \rightarrow \infty} d_{k}\left(x_{n}\right.$, $x)=\lim _{n \rightarrow \infty} d_{k}\left(x, x_{n}\right)=0$.

(ii) $\left\{x_{n}\right\}$ is called Cauchy in $X$, if $\lim _{n, m \rightarrow \infty} d_{k}\left(x_{n}, x_{m}\right)=$ $\lim _{n, m \rightarrow \infty} d_{k}\left(x_{m}, x_{n}\right)=0$.

(iii) $\left(X, d_{k}\right)$ is called complete if every Cauchy sequence in $X$ is convergent in $X$.

Definition 13 (see [6]). Let $\mathrm{X}$ be nonempty set, $G$ and $H$ are subsets of $X$. A function $T: G \cup H \longrightarrow G \cup H$ is called a cyclic map if $T(G) \subseteq H$ and $T(H) \subseteq G$.

Definition 14 (see [17]). Let $\left(X, d_{k}\right)$ be a dislocated quasi extended $b$-metric space, $G$ and $H$ be subsets of $X$. A function $T: G \cup H \longrightarrow G \cup H$ is called dqeb-cyclic weakly contraction if there exists continuous and non-decreasing function $\varphi$ : $[0, \infty) \longrightarrow[0, \infty)$ such that for every $x \in G, y \in H$,

$$
k(x, y) d_{k}(T x, T y) \leq d_{k}(x, y)-\varphi\left(d_{k}(x, y)\right),
$$

where $\varphi(t)=0$ if and only if $t=0$.

Definition 15 (see [20]). Let $\left(X, d_{k}\right)$ be a dislocated quasi extended $b$-metric space, $G$ and $H$ be subsets of $X$. A function $T: G \cup H \longrightarrow G \cup H$ is called a cyclic $\varphi$ contraction if $T$ is a cyclic and there exists a continuous and non-decreasing function $\varphi:[0, \infty) \longrightarrow[0, \infty)$ such that for every $x \in G$, $y \in H$

$$
d_{k}(T x, T y) \leq \varphi\left(d_{k}(x, y)\right)
$$

\section{Main Results}

In this section, we show some theorems and examples of the existence and uniqueness of fixed point for generalized dqebcyclic weakly contraction mapping in complete dislocated quasi extended $b$-metric space.

Theorem 16. Let $\left(X, d_{k}\right)$ be a complete dislocated quasi extended b-metric space, $G$ and $H$ be closed subsets of $X$. If $T: G \cup H \longrightarrow G \cup H$ is a cyclic map that satisfies the condition of dqeb-cyclic weakly contraction and $\lim _{n, m \rightarrow \infty} k\left(x_{n}, x_{m}\right)=$ $L>0$, then $T$ has a unique fixed point in $G \cap H$.

Proof. Since $T$ is a cyclic map, if taking $x_{0} \in G$, then $T x_{0} \in H$ and $T^{2} x_{0} \in G$. Define a sequence $\left\{x_{n}\right\}$, where $x_{n}=T x_{n-1}=$ $T^{n} x_{0}$. So we have $x_{2 n} \in G$ and $x_{2 n-1} \in H$ for $n=1,2,3 \ldots$

Since $k(x, y) \geq 1$ for all $x, y \in X$, then for all $n \in N$ we have

$$
\begin{aligned}
d_{k}\left(x_{n+1}, x_{n}\right) & \leq k\left(x_{n+1}, x_{n}\right) d_{k}\left(x_{n+1}, x_{n}\right) \\
& =k\left(x_{n+1}, x_{n}\right) d_{k}\left(T x_{n}, T x_{n-1}\right) \\
& \leq d_{k}\left(x_{n}, x_{n-1}\right)-\varphi\left(d_{k}\left(x_{n}, x_{n-1}\right)\right) \\
& \leq d_{k}\left(x_{n}, x_{n-1}\right) .
\end{aligned}
$$

Thus we have $\left\{d_{k}\left(x_{n+1}, x_{n}\right)\right\}$ is a nonincreasing sequence of non-negative real numbers.

Claim that $\lim _{n \rightarrow \infty} d_{k}\left(x_{n+1}, x_{n}\right)=0$. Suppose $\lim _{n \rightarrow \infty} d_{k}\left(x_{n+1}, x_{n}\right)=\beta$.

Since $\varphi$ is nondecreasing and $k\left(x_{n+1}, x_{n}\right) \geq 1$, we have

$$
\begin{aligned}
d_{k}\left(x_{n+1}, x_{n}\right) & \leq k\left(x_{n+1}, x_{n}\right) d_{k}\left(x_{n+1}, x_{n}\right) \\
& \leq d_{k}\left(x_{n}, x_{n-1}\right)-\varphi\left(d_{k}\left(x_{n}, x_{n-1}\right) .\right.
\end{aligned}
$$

Since $\varphi$ is continuous then for $n \longrightarrow \infty$, we have $\beta \leq \beta-\varphi(\beta)$. Since $\varphi \geq 0$, thus we get $\varphi(\beta)=0$. Hence we have $\beta=0$. Similarly we have $\lim _{n \rightarrow \infty} d_{k}\left(x_{n}, x_{n+1}\right)=0$. $X$.

Now, we have to prove that $\left\{x_{n}\right\}$ is a Cauchy sequence in

Suppose $\left\{x_{n}\right\}$ is not a Cauchy sequence. Then there exists $\varepsilon>0$ such that every $n$, there exists $n_{k}, m_{k}>n$ such that $d_{k}\left(n_{k}, m_{k}\right) \geq \varepsilon$ and $d_{k}\left(n_{k-1}, m_{k}\right)<\varepsilon$.

From (11) we have

$$
\begin{aligned}
& k\left(n_{k-1}, m_{k-1}\right) d_{k}\left(n_{k}, m_{k}\right) \\
& \quad=k\left(n_{k-1}, m_{k-1}\right) d_{k}\left(T n_{k-1}, T m_{k-1}\right) \\
& \quad \leq d_{k}\left(n_{k-1}, m_{k-1}\right)-\varphi\left(d_{k}\left(n_{k-1}, m_{k-1}\right)\right) \\
& \quad \leq d_{k}\left(n_{k-1}, m_{k-1}\right) .
\end{aligned}
$$

This implies

$$
d_{k}\left(n_{k-1}, m_{k-1}\right) \geq \varepsilon k\left(n_{k-1}, m_{k-1}\right) .
$$

We also have that

$$
\begin{aligned}
d_{k} & \left(n_{k-1}, m_{k-1}\right) \\
& \leq k\left(n_{k-1}, m_{k-1}\right)\left(d_{k}\left(n_{k-1}, m_{k}\right)+d_{k}\left(m_{k}, m_{k-1}\right)\right) \\
& <\varepsilon k\left(n_{k-1}, m_{k-1}\right)+k\left(n_{k-1}, m_{k-1}\right) d_{k}\left(m_{k}, m_{k-1}\right) .
\end{aligned}
$$


It implies $\lim _{k \rightarrow \infty} d_{k}\left(n_{k-1}, m_{k-1}\right) \leq \varepsilon \lim _{k \longrightarrow \infty} k\left(n_{k-1}, m_{k-1}\right)$. Therefore we have $\varepsilon \lim _{k \rightarrow \infty} k\left(n_{k-1}, m_{k-1}\right) \leq \lim _{k \rightarrow \infty} d_{k}\left(n_{k-1}\right.$, $\left.m_{k-1}\right) \leq \varepsilon \lim _{k \rightarrow \infty} k\left(n_{k-1}, m_{k-1}\right)$, so we have $\varepsilon L \leq$ $\lim _{k \rightarrow \infty} d_{k}\left(n_{k-1}, m_{k-1}\right) \leq \varepsilon L$, thus we obtain $\lim _{k \rightarrow \infty} d_{k}\left(n_{k-1}\right.$, $\left.m_{k-1}\right)=\varepsilon L$. From (15) and (16) we have

$$
\begin{aligned}
& k\left(n_{k-1}, m_{k-1}\right) d_{k}\left(n_{k}, m_{k}\right) \\
& \quad \leq d_{k}\left(n_{k-1}, m_{k-1}\right)-\varphi\left(d_{k}\left(n_{k-1}, m_{k-1}\right)\right) \\
& \quad \leq d_{k}\left(n_{k-1}, m_{k-1}\right) \\
& \varepsilon k\left(n_{k-1}, m_{k-1}\right) \\
& \quad \leq d_{k}\left(n_{k-1}, m_{k-1}\right)-\varphi\left(d_{k}\left(n_{k-1}, m_{k-1}\right)\right) \\
& \quad \leq d_{k}\left(n_{k-1}, m_{k-1}\right) .
\end{aligned}
$$

For $k \longrightarrow \infty$ and using continuity of $\varphi$, we get

$$
\varepsilon L \leq \varepsilon L-\varphi(\varepsilon L) \leq \varepsilon L
$$

Since $k(x, y) \geq 1$ and $\lim _{n, m \rightarrow \infty} k\left(x_{n}, x_{m}\right)=L$, we have $L \geq 1$. Thus we have $\varphi(\varepsilon L)=0$, this implies $\varepsilon L=0$. Since $\varepsilon>0$ then we obtain $L=0$, which is a contradiction.

Hence $\left\{x_{n}\right\}$ is a Cauchy sequence in $X$. Since $X$ complete, there exists $x^{*} \in X$ such that $d_{k}\left(x_{n}, x^{*}\right) \longrightarrow 0$ for $n \longrightarrow \infty$. Similarly we can have $d_{k}\left(x_{n}, x^{*}\right) \longrightarrow 0$.

Since the sequence $\left\{x_{2 n}\right\} \in G,\left\{x_{2 n-1}\right\} \in H$ and $G, H$ be closed, then we have $x^{*} \in G \cap H$.

Now we prove that $x^{*}$ is a fixed point of T. Using (2) and (11) we have

$$
\begin{aligned}
& d_{k}\left(T x^{*}, x^{*}\right) \leq k\left(T x^{*}, x^{*}\right) \\
& \cdot\left(d\left(T x^{*}, T x_{n-1}\right)+d\left(T x_{n-1}, x^{*}\right)\right) \leq k\left(T x^{*}, x^{*}\right) \\
& \cdot\left(d\left(x^{*}, x_{n-1}\right)-\varphi\left(d\left(x^{*}, x_{n-1}\right)\right)+d\left(x_{n}, x^{*}\right)\right) .
\end{aligned}
$$

Using continuity of $\varphi$ and for $n \longrightarrow \infty$, we have $d_{k}\left(T x^{*}, x^{*}\right) \leq$ $-k\left(T x^{*}, x^{*}\right) \varphi(0) \leq 0$.

Thus $d_{k}\left(T x^{*}, x^{*}\right)=0$, hence $T x^{*}=x^{*}$.

Now we have to show that $T$ has unique fixed point in $X$. Suppose that $u$ is an another fixed point of $T$,

$$
\begin{aligned}
d_{k}\left(x^{*}, u\right) & =d_{k}\left(T x^{*}, T u\right) \leq k\left(x^{*}, u\right) d_{k}\left(T x^{*}, T u\right) \\
& \leq d_{k}\left(x^{*}, u\right)-\varphi\left(d_{k}\left(x^{*}, u\right)\right) .
\end{aligned}
$$

Thus we get $\varphi\left(d_{k}\left(x^{*}, u\right)\right) \leq 0$. Since $\varphi \geq 0$, we have $\varphi\left(d_{k}\left(x^{*}, u\right)\right)=0$. Which implies that $d_{k}\left(x^{*}, u\right)=0$, so we have $x^{*}=u$.

Example 17. Let $X=[-1,1]$ and $\left(X, d_{k}\right)$ be a dislocated quasi extended $b$-metric space which in Example 8. Let $T: G \cup$ $H \longrightarrow G \cup H$ be a function defined by $T x=-x / 2$, where $G=$ $[-1,0], H=[0,1]$. Let $\varphi:[0, \infty) \longrightarrow[0, \infty)$ be a function which is defined by $\varphi(t)=t / 4$

In fact, It is clear that $T$ is a cyclic map, indeed $T(G) \subseteq H$ and $T(H) \subseteq G$.
Now, we have to show that

$$
\begin{aligned}
& k(x, y) d_{k}(T x, T y) \leq d_{k}(x, y)-\varphi\left(d_{k}(x, y)\right) \\
& k(x, y) d_{k}(T x, T y)=\frac{2+|x y|}{2} d_{k}\left(\frac{-x}{2}, \frac{-y}{2}\right) \\
& =\frac{2+|x y|}{2}\left[\left(\left|\frac{-x}{2}\right|+\left|\frac{-y}{2}\right|\right)+\frac{|-x / 2|^{2}}{5}\right. \\
& \left.+\frac{|-y / 2|^{2}}{6}\right]=\frac{2+|x y|}{4}\left[|x|+|y|+\frac{|x|^{2}}{10}+\frac{|y|^{2}}{12}\right] \\
& =\frac{1}{4}\left[\left(2(|x|+|y|)+\frac{|x|^{2}}{10}+\frac{|y|^{2}}{12}\right)\right. \\
& \left.+|x y|\left((|x|+|y|)+\frac{|x|^{2}}{10}+\frac{|y|^{2}}{12}\right)\right] \\
& \leq \frac{1}{4}\left[\left(2(|x|+|y|)+\frac{|x|^{2}}{10}+\frac{|y|^{2}}{12}\right)\right. \\
& \left.+\left((|x|+|y|)+\frac{|x|^{2}}{10}+\frac{|y|^{2}}{12}\right)\right] \\
& \left.\leq \frac{1}{4}\left[3(|x|+|y|)+\frac{|x|^{2}}{5}+\frac{|y|^{2}}{6}\right)\right] \leq \frac{3}{4}(|x| \\
& \left.+|y|+\frac{|x|^{2}}{10}+\frac{|y|^{2}}{12}\right) \leq \frac{3}{4}\left(|x|+|y|+\frac{|x|^{2}}{5}\right. \\
& \left.+\frac{|y|^{2}}{6}\right)=\left(|x|+|y|+\frac{|x|^{2}}{5}+\frac{|y|^{2}}{6}\right)-\frac{1}{4}(|x| \\
& \left.+|y|+\frac{|x|^{2}}{5}+\frac{|y|^{2}}{6}\right)=d_{k}(x, y)-\varphi\left(d_{k}(x, y)\right) \text {. }
\end{aligned}
$$

Hence, $T$ has a deqb-weak contraction property of Theorem 16 and $x=0$ is the unique fixed point of $T$.

Theorem 18. Let $\left(X, d_{k}\right)$ be a complete dislocated quasi extended b-metric space, $G$ and $H$ be closed subsets of $X$. If $T: G \cup H \longrightarrow G \cup H$ is a cyclic map, continuous mapping and $\lim _{n, m \rightarrow \infty} k\left(x_{n}, x_{m}\right)=L>0$, such that

$$
k(x, y) d_{k}(T x, T y) \leq d_{k}(x, y)-\varphi\left(d_{k}(T x, T y)\right)
$$

where $\varphi:[0, \infty) \longrightarrow[0, \infty)$ is a nondecreasing, continuous mapping and $\varphi(t)=0$ iff $t=0$.

Then $T$ has a unique fixed point in $G \cap H$.

Proof. Since $T$ is a cyclic map, if taking $x_{0} \in G$, then $T x_{0} \in H$ and $T^{2} x_{0} \in G$. Define a sequence $\left\{x_{n}\right\}$, where $x_{n}=T x_{n-1}=$ $T^{n} x_{0}$. So we have $x_{2 n} \in G$ and $x_{2 n-1} \in H$ for $n=1,2,3 \ldots$ 
By using (23) and for all $n \in N$, we have

$$
\begin{aligned}
d_{k}\left(x_{n+1}, x_{n}\right) & \leq k\left(x_{n+1}, x_{n}\right) d_{k}\left(x_{n+1}, x_{n}\right) \\
& =k\left(x_{n+1}, x_{n}\right) d_{k}\left(T x_{n}, T x_{n-1}\right) \\
& \leq d_{k}\left(x_{n}, x_{n-1}\right)-\varphi\left(d_{k}\left(T x_{n}, T x_{n-1}\right)\right) \\
& \leq d_{k}\left(x_{n}, x_{n-1}\right) .
\end{aligned}
$$

Thus we have $\left\{d_{k}\left(x_{n+1}, x_{n}\right)\right\}$ is a nonincreasing seq-uence of non-negative real numbers. Claim that $\lim _{n \rightarrow \infty} d_{k}\left(x_{n+1}\right.$, $\left.x_{n}\right)=0$. Suppose $\lim _{n \longrightarrow \infty} d_{k}\left(x_{n+1}, x_{n}\right)=\beta$.

Since $\varphi$ is non-decreasing and $k\left(x_{n+1}, x_{n}\right) \geq 1$, then we have

$$
\begin{aligned}
d_{k}\left(x_{n+1}, x_{n}\right) & \leq k\left(x_{n+1}, x_{n}\right) d_{k}\left(x_{n+1}, x_{n}\right) \\
& \leq d_{k}\left(x_{n}, x_{n-1}\right)-\varphi\left(d_{k}\left(T x_{n}, T x_{n-1}\right)\right) \\
& \leq d_{k}\left(x_{n}, x_{n-1}\right)-\varphi\left(d_{k}\left(T x_{n}, T x_{n-1}\right)\right) \\
& =d_{k}\left(x_{n}, x_{n-1}\right)-\varphi\left(d_{k}\left(x_{n+1}, x_{n}\right)\right) .
\end{aligned}
$$

Since $\varphi$ is a continuous mapping then for $n \longrightarrow \infty$, we have $\beta \leq \beta-\varphi(\beta)$. Since $\varphi \geq 0$, thus we get $\varphi(\beta)=0$. Hence we have $\beta=0$. Similarly we have $\lim _{n \longrightarrow \infty} d_{k}\left(x_{n}, x_{n+1}\right)=0$. $X$.

Now, we have to prove that $\left\{x_{n}\right\}$ is a Cauchy sequence in

Suppose $\left\{x_{n}\right\}$ is not a Cauchy, then there exists $\varepsilon>0$ such that every $n$, there exists $n_{k}, m_{k}>n$ such that

$$
\begin{aligned}
d_{k}\left(n_{k}, m_{k}\right) & \geq \varepsilon \\
\text { and } d_{k}\left(n_{k-1}, m_{k}\right) & <\varepsilon .
\end{aligned}
$$

From (23) we have

$$
\begin{aligned}
k & \left(n_{k-1}, m_{k-1}\right) d_{k}\left(n_{k}, m_{k}\right) \\
& =k\left(n_{k-1}, m_{k-1}\right) d_{k}\left(T n_{k-1}, T m_{k-1}\right) \\
& \leq d_{k}\left(n_{k-1}, m_{k-1}\right)-\varphi\left(d_{k}\left(T n_{k-1}, T m_{k-1}\right)\right) \\
& \leq d_{k}\left(n_{k-1}, m_{k-1}\right) .
\end{aligned}
$$

By using (26) and (27), then we have

$$
d_{k}\left(n_{k-1}, m_{k-1}\right) \geq \varepsilon k\left(n_{k-1}, m_{k-1}\right) .
$$

By using (2) and (26) we also have that

$$
\begin{aligned}
d_{k} & \left(n_{k-1}, m_{k-1}\right) \\
& \leq k\left(n_{k-1}, m_{k-1}\right)\left(d_{k}\left(n_{k-1}, m_{k}\right)+d_{k}\left(m_{k}, m_{k-1}\right)\right) \\
& <\varepsilon k\left(n_{k-1}, m_{k-1}\right)+k\left(n_{k-1}, m_{k-1}\right) d_{k}\left(m_{k}, m_{k-1}\right) .
\end{aligned}
$$

It implies $\lim _{k \rightarrow \infty} d_{k}\left(n_{k-1}, m_{k-1}\right) \leq \varepsilon \lim _{k \longrightarrow \infty} k\left(n_{k-1}, m_{k-1}\right)$. By using (28) and (29), we have $\varepsilon \lim _{k \rightarrow \infty} k\left(n_{k-1}, m_{k-1}\right) \leq$ $\lim _{k \rightarrow \infty} d_{k}\left(n_{k-1}, m_{k-1}\right) \leq \varepsilon \lim _{k \rightarrow \infty} k\left(n_{k-1}, m_{k-1}\right)$, so we get $\varepsilon L \leq \lim _{k \rightarrow \infty} d_{k}\left(n_{k-1}, m_{k-1}\right) \leq \varepsilon L$, thus we obtain $\lim _{k \rightarrow \infty} d_{k}\left(n_{k-1}, m_{k-1}\right)=\varepsilon L$.
From (23) and (28) we have

$$
\begin{aligned}
& k\left(n_{k-1}, m_{k-1}\right) d_{k}\left(n_{k}, m_{k}\right) \\
& \quad \leq d_{k}\left(n_{k-1}, m_{k-1}\right)-\varphi\left(d_{k}\left(n_{k}, m_{k}\right)\right) \\
& \quad \leq d_{k}\left(n_{k-1}, m_{k-1}\right) .
\end{aligned}
$$

Since $\varphi$ is a non-decreasing and $\varphi \geq 0$, we have

$$
\begin{aligned}
\varepsilon k\left(n_{k-1}, m_{k-1}\right) \leq & d_{k}\left(n_{k-1}, m_{k-1}\right)-\varphi\left(d_{k}\left(n_{k}, m_{k}\right)\right) \\
\leq & d_{k}\left(n_{k-1}, m_{k-1}\right) \\
& -\varphi\left(\varepsilon k\left(n_{k-1}, m_{k-1}\right)\right) \\
\leq & d_{k}\left(n_{k-1}, m_{k-1}\right) .
\end{aligned}
$$

For $k \longrightarrow \infty$ and using continuity of $\varphi$, we get

$$
\varepsilon L \leq \varepsilon L-\varphi(\varepsilon L) \leq \varepsilon L
$$

Since $k(x, y) \geq 1$ and $\lim _{n, m \rightarrow \infty} k\left(x_{n}, x_{m}\right)=L$, thus we have $L \geq 1$. However, from (32) we have $\varphi(\varepsilon L)=0$, this implies $\varepsilon L=0$. Since $\varepsilon>0$ then we obtain $L=0$ which is a contradiction.

Hence $\left\{x_{n}\right\}$ is a Cauchy sequence in $X$.

Since $X$ complete, there exists $x^{*} \in X$ such that $d_{k}\left(x_{n}, x^{*}\right) \longrightarrow 0$ and $d_{k}\left(x^{*}, x_{n}\right) \longrightarrow 0$ for $n \longrightarrow \infty$.

Since the sequence $\left\{x_{2 n}\right\} \in G,\left\{x_{2 n-1}\right\} \in H$ and $G, H$ closed, we have $x^{*} \in G \cap H$.

Now we have to prove that $x^{*}$ is a fixed point of $T$. By using (2) and (23), we have

$$
\begin{aligned}
& d_{k}\left(T x^{*}, x^{*}\right) \leq k\left(T x^{*}, x^{*}\right) \\
& \cdot\left(d\left(T x^{*}, T x_{n-1}\right)+d\left(T x_{n-1}, x^{*}\right)\right)=k\left(T x^{*}, x^{*}\right) \\
& \cdot\left(d\left(T x^{*}, T x_{n-1}\right)+d\left(x_{n}, x^{*}\right)\right) \leq k\left(T x^{*}, x^{*}\right) \\
& \cdot\left(k\left(x^{*}, x_{n-1}\right) d\left(T x^{*}, T x_{n-1}\right)+d\left(x_{n}, x^{*}\right)\right) \\
& \leq k\left(T x^{*}, x^{*}\right) \\
& \cdot\left(d\left(x^{*}, x_{n-1}\right)-\varphi\left(d\left(T x^{*}, T x_{n-1}\right)\right)+d\left(x_{n}, x^{*}\right)\right) \\
& \leq k\left(T x^{*}, x^{*}\right)\left(d\left(x^{*}, x_{n-1}\right)+d\left(x_{n}, x^{*}\right)\right) .
\end{aligned}
$$

Thus for $n \longrightarrow \infty$, we have $d_{k}\left(T x^{*}, x^{*}\right)=0$, hence $T x^{*}=x^{*}$.

Now we have to show that $T$ has unique fixed point in $X$. Suppose that $u$ is an another fixed point $T$,

$$
\begin{aligned}
d_{k}\left(x^{*}, u\right) & =d_{k}\left(T x^{*}, T u\right) \leq k\left(x^{*}, u\right) d_{k}\left(T x^{*}, T u\right) \\
& \leq d_{k}\left(x^{*}, u\right)-\varphi\left(d_{k}\left(T x^{*}, T u\right)\right) \\
& =d_{k}\left(x^{*}, u\right)-\varphi\left(d_{k}\left(x^{*}, u\right)\right) .
\end{aligned}
$$

Thus we get $\varphi\left(d_{k}\left(x^{*}, u\right)\right) \leq 0$. Since $\varphi \geq 0$, we have $\varphi\left(d_{k}\left(x^{*}, u\right)\right)=0$. Which implies that $d_{k}\left(x^{*}, u\right)=0$, so we have $x^{*}=u$. 
Example 19. Let $X=[-1,1]$ and $\left(X, d_{k}\right)$ be a dislocated quasi extended $b$-metric space which in Example 8. Let $T: G \cup$ $H \longrightarrow G \cup H$ be a function defined by $T x=-x / 2$, where $G=$ $[-1,0], H=[0,1]$. Let $\varphi:[0, \infty) \longrightarrow[0, \infty)$ be a function and defined as, $\varphi(t)=t / 8$.

In fact, it is clear that $T$ is cyclic map, indeed $T(G) \subseteq H$ and $T(H) \subseteq G$.

Now, for all $x, y \in X$ we have to show that

$$
\begin{aligned}
& k(x, y) d_{k}(T x, T y) \leq d_{k}(x, y)-\varphi\left(d_{k}(T x, T y)\right) . \\
& k(x, y) d_{k}(T x, T y)=\frac{2+|x y|}{2} d_{k}\left(\frac{-x}{2}, \frac{-y}{2}\right) \\
& =\frac{2+|x y|}{2}\left[\left(\left|\frac{-x}{2}\right|+\left|\frac{-y}{2}\right|\right)+\frac{|-x / 2|^{2}}{5}\right. \\
& \left.+\frac{|-y / 2|^{2}}{6}\right]=\frac{2+|x y|}{4}\left[|x|+|y|+\frac{|x|^{2}}{10}+\frac{|y|^{2}}{12}\right] \\
& =\frac{1}{4}\left[\left(2(|x|+|y|)+\frac{|x|^{2}}{10}+\frac{|y|^{2}}{12}\right)\right. \\
& \left.+|x y|\left((|x|+|y|)+\frac{|x|^{2}}{10}+\frac{|y|^{2}}{12}\right)\right] \\
& \leq \frac{1}{4}\left[\left(2(|x|+|y|)+\frac{|x|^{2}}{10}+\frac{|y|^{2}}{12}\right)\right. \\
& \left.+\left((|x|+|y|)+\frac{|x|^{2}}{10}+\frac{|y|^{2}}{12}\right)\right]=\frac{1}{4}[3(|x|+|y|) \\
& \left.\left.+\frac{|x|^{2}}{5}+\frac{|y|^{2}}{6}\right)\right] \leq \frac{3}{4}\left(|x|+|y|+\frac{|x|^{2}}{5}+\frac{|y|^{2}}{6}\right) \\
& =\left(|x|+|y|+\frac{|x|^{2}}{5}+\frac{|y|^{2}}{6}\right)-\frac{1}{4}(|x|+|y| \\
& \left.+\frac{|x|^{2}}{5}+\frac{|y|^{2}}{6}\right) \leq\left(|x|+|y|+\frac{|x|^{2}}{5}+\frac{|y|^{2}}{6}\right) \\
& -\frac{1}{16}\left(|x|+|y|+\frac{|x|^{2}}{5}+\frac{|y|^{2}}{6}\right)=(|x|+|y| \\
& \left.+\frac{|x|^{2}}{5}+\frac{|y|^{2}}{6}\right)-\frac{1}{8}\left(\frac{|x|}{2}+\frac{|y|}{2}+\frac{|x|^{2}}{10}+\frac{|y|^{2}}{12}\right) \\
& \leq\left(|x|+|y|+\frac{|x|^{2}}{5}+\frac{|y|^{2}}{6}\right)-\frac{1}{8}\left(\frac{|x|}{2}+\frac{|y|}{2}\right. \\
& \left.+\frac{|x|^{2}}{20}+\frac{|y|^{2}}{24}\right)=\left(|x|+|y|+\frac{|x|^{2}}{5}+\frac{|y|^{2}}{6}\right)
\end{aligned}
$$

Hence, $T$ has a deqb-weak contraction property of Theorem 18 and $x=0$ is the unique fixed point of $T$.

Theorem 20. Let $\left(X, d_{k}\right)$ be a complete dislocated quasi extended b-metric space, $G$ and $H$ be closed subsets of $X$. If $T: G \cup H \longrightarrow G \cup H$ is a cyclic, continuous mapping and $\lim _{n, m \rightarrow \infty} k\left(x_{n}, x_{m}\right)=L>0$, such that

$$
k(T x, T y) d_{k}(T x, T y) \leq d_{k}(x, y)-\varphi\left(d_{k}(x, y)\right),
$$

where $\varphi:[0, \infty) \longrightarrow[0, \infty)$ be a nondecreasing, continuous function and $\varphi(t)=0$ iff $t=0$.

Then $T$ has a unique fixed point in $G \cap H$.

Proof. Since $T$ is a cyclic map, taking $x_{0} \in G$, then $T x_{0} \in H$ and $T^{2} x_{0} \in G$. Define a sequence $\left\{x_{n}\right\}$, where $x_{n}=T x_{n-1}=$ $T^{n} x_{0}$. So we have $x_{2 n} \in G$ and $x_{2 n-1} \in H$ for $n=1,2,3 \ldots$

From (37), then for all $n \in N$ we have

$$
\begin{aligned}
d_{k}\left(x_{n+1}, x_{n}\right) & \leq k\left(x_{n+1}, x_{n}\right) d_{k}\left(x_{n+1}, x_{n}\right) \\
& =k\left(T x_{n}, T x_{n-1}\right) d_{k}\left(T x_{n}, T x_{n-1}\right) \\
& \leq d_{k}\left(x_{n}, x_{n-1}\right)-\varphi\left(d_{k}\left(x_{n}, x_{n-1}\right)\right) \\
& \leq d_{k}\left(x_{n}, x_{n-1}\right) .
\end{aligned}
$$

Thus we have $\left\{d_{k}\left(x_{n+1}, x_{n}\right)\right\}$ be a nonincreasing seq-uence of non-negative real numbers. Claim that $\lim _{n \rightarrow \infty} d_{k}\left(x_{n+1}\right.$, $\left.x_{n}\right)=0$. Suppose $\lim _{n \rightarrow \infty} d_{k}\left(x_{n+1}, x_{n}\right)=\beta$.

Since $\varphi$ is a nondecreasing and $k\left(x_{n+1}, x_{n}\right) \geq 1$, then we have

$$
\begin{aligned}
d_{k}\left(x_{n+1}, x_{n}\right) & \leq k\left(x_{n+1}, x_{n}\right) d_{k}\left(x_{n+1}, x_{n}\right) \\
& \leq d_{k}\left(x_{n}, x_{n-1}\right)-\varphi\left(d_{k}\left(x_{n}, x_{n-1}\right)\right) .
\end{aligned}
$$

Since $\varphi$ is continuous then for $\longrightarrow \infty$, we have $\beta \leq \beta-\varphi(\beta)$. Since $\varphi \geq 0$, thus we get $\varphi(\beta)=0$. Hence we have $\beta=0$. Similarly we have $\lim _{n \rightarrow \infty} d_{k}\left(x_{n}, x_{n+1}\right)=0$. $X$.

Now, we have to prove that $\left\{x_{n}\right\}$ is a Cauchy sequence in

Suppose $\left\{x_{n}\right\}$ is not a Cauchy, then there exists $\varepsilon>0$ such that every $n$, there exists $n_{k}, m_{k}>n$ such that $d_{k}\left(n_{k}, m_{k}\right) \geq \varepsilon$ and $d_{k}\left(n_{k-1}, m_{k}\right)<\varepsilon$.

By using (37) we have

$$
\begin{aligned}
& k\left(n_{k}, m_{k}\right) d_{k}\left(n_{k}, m_{k}\right) \\
& \quad=k\left(T n_{k-1}, T m_{k-1}\right) d_{k}\left(T n_{k-1}, T m_{k-1}\right) \\
& \quad \leq d_{k}\left(n_{k-1}, m_{k-1}\right)-\varphi\left(d_{k}\left(n_{k-1}, m_{k-1}\right)\right) \\
& \quad \leq d_{k}\left(n_{k-1}, m_{k-1}\right) .
\end{aligned}
$$


Since $d_{k}\left(n_{k}, m_{k}\right) \geq \varepsilon$, we get

$$
d_{k}\left(n_{k-1}, m_{k-1}\right) \geq \varepsilon k\left(n_{k}, m_{k}\right) .
$$

By using (2), we also have that

$$
\begin{aligned}
& d_{k}\left(n_{k-1}, m_{k-1}\right) \\
& \quad \leq k\left(n_{k-1}, m_{k-1}\right)\left(d_{k}\left(n_{k-1}, m_{k}\right)+d_{k}\left(m_{k}, m_{k-1}\right)\right) \\
& \quad \leq \varepsilon k\left(n_{k-1}, m_{k-1}\right)+k\left(n_{k-1}, m_{k-1}\right) d_{k}\left(m_{k}, m_{k-1}\right) .
\end{aligned}
$$

It implies that $\lim _{k \rightarrow \infty} d_{k}\left(n_{k-1}, m_{k-1}\right) \leq \varepsilon \lim _{k \rightarrow \infty} k\left(n_{k-1}\right.$, $\left.m_{k-1}\right)$.

Therefore, from (40) and (41), we have

$$
\begin{aligned}
\varepsilon \lim _{k \rightarrow \infty} k\left(n_{k}, m_{k}\right) & \leq \lim _{k \rightarrow \infty} d_{k}\left(n_{k-1}, m_{k-1}\right) \\
& \leq \varepsilon \lim _{k \rightarrow \infty} k\left(n_{k-1}, m_{k-1}\right) .
\end{aligned}
$$

Thus we have $\varepsilon L \leq \lim _{k \rightarrow \infty} d_{k}\left(n_{k-1}, m_{k-1}\right) \leq \varepsilon L$, and we obtain

$$
\lim _{k \longrightarrow \infty} d_{k}\left(n_{k-1}, m_{k-1}\right)=\varepsilon L
$$

From (40) and (44) and $d_{k}\left(n_{k}, m_{k}\right) \geq \varepsilon$, we have

$$
\begin{aligned}
& \varepsilon k\left(n_{k}, m_{k}\right) \leq k\left(n_{k}, m_{k}\right) d_{k}\left(n_{k}, m_{k}\right) \\
& \quad \leq d_{k}\left(n_{k-1}, m_{k-1}\right)-\varphi\left(d_{k}\left(n_{k-1}, m_{k-1}\right)\right) \\
& \quad \leq d_{k}\left(n_{k-1}, m_{k-1}\right) .
\end{aligned}
$$

By using (45) and continuity of $\varphi$, then for $k \longrightarrow \infty$ we get

$$
\varepsilon L \leq \varepsilon L-\varphi(\varepsilon L) \leq \varepsilon L
$$

Since $k(x, y) \geq 1$ and $\lim _{n, m \rightarrow \infty} k\left(x_{n}, x_{m}\right)=L$, thus we have $L \geq 1$. However, from (46) we have $\varphi(\varepsilon L)=0$, this implies $\varepsilon L=0$ and since $\varepsilon>0$ then we obtain $L=0$ which is a contradiction.

Hence $\left\{x_{n}\right\}$ is a Cauchy sequence in $X$.

Since $X$ complete, there exists $x^{*} \in X$ such that $d_{k}\left(x_{n}, x^{*}\right) \longrightarrow 0$ for $n \longrightarrow \infty$. Since the sequence $\left\{x_{2 n}\right\} \in G$, $\left\{x_{2 n-1}\right\} \in H$ and $G, H$ closed, it implies that $x^{*} \in G \cap H$.

Now, we have to prove that $x^{*}$ is a fixed point of $T$.

$$
\begin{aligned}
& d_{k}\left(T x^{*}, x^{*}\right) \leq k\left(T x^{*}, x^{*}\right) \\
& \cdot\left(d\left(T x^{*}, T x_{n-1}\right)+d\left(T x_{n-1}, x^{*}\right)\right)=k\left(T x^{*}, x^{*}\right) \\
& \cdot\left(d\left(T x^{*}, T x_{n-1}\right)+d\left(x_{n}, x^{*}\right)\right) \leq k\left(T x^{*}, x^{*}\right) \\
& \cdot\left(k\left(T x^{*}, T x_{n-1}\right) d\left(T x^{*}, T x_{n-1}\right)+d\left(x_{n}, x^{*}\right)\right) \\
& \leq k\left(T x^{*}, x^{*}\right) \\
& \cdot\left(d\left(x^{*}, x_{n-1}\right)-\varphi\left(d\left(x^{*}, x_{n-1}\right)\right)+d\left(x_{n}, x^{*}\right)\right) \\
& \leq k\left(T x^{*}, x^{*}\right)\left(d\left(x^{*}, x_{n-1}\right)+d\left(x_{n}, x^{*}\right)\right) .
\end{aligned}
$$

Thus for $n \longrightarrow \infty$, we have $d_{k}\left(T x^{*}, x^{*}\right)=0$, hence $T x^{*}=x^{*}$.
Now we have to show that $T$ has unique fixed point in $X$. Suppose $u$ is an another fixed point of $T$,

$$
\begin{aligned}
d_{k}\left(x^{*}, u\right) & =d_{k}\left(T x^{*}, T u\right) \\
& \leq k\left(T x^{*}, T u\right) d_{k}\left(T x^{*}, T u\right) \\
& \leq d_{k}\left(x^{*}, u\right)-\varphi\left(d_{k}\left(x^{*}, u\right)\right) .
\end{aligned}
$$

Thus we get $\varphi\left(d_{k}\left(x^{*}, u\right)\right) \leq 0$. Since $\varphi \geq 0$, we have $\varphi\left(d_{k}\left(x^{*}, u\right)\right)=0$. Which implies that $d_{k}\left(x^{*}, u\right)=0$, so we have $x^{*}=u$.

Example 21. Let $X=[-1,1]$ and $\left(\mathrm{X}, \mathrm{d}_{\mathrm{k}}\right)$ be a dislocated quasi extended $b$-metric space which in Example 8. Let $T: G \cup$ $H \longrightarrow G \cup H$ be a function defined by $T x=-x / 2$, where $G=[-1,0], H=[0,1]$, and let $\varphi:[0, \infty) \longrightarrow[0, \infty)$ be a function and defined as, $\varphi(t)=(7 / 16) t$.

In fact it clear $T$ is cyclic, since $T(G) \subseteq H$ and $T(H) \subseteq G$.

Now, we have to show that

$$
\begin{aligned}
& k(T x, T y) d_{k}(T x, T y) \leq d_{k}(x, y)-\varphi\left(d_{k}(x, y)\right) \\
& k(T x, T y) d_{k}(T x, T y)=k\left(\frac{-x}{2}, \frac{-y}{2}\right) d_{k}\left(\frac{-x}{2}, \frac{-y}{2}\right) \\
& \quad=\frac{8+|x y|}{8}\left[\left(\left|\frac{-x}{2}\right|+\left|\frac{-y}{2}\right|\right)+\frac{|-x / 2|^{2}}{5}\right. \\
&\left.+\frac{|-y / 2|^{2}}{6}\right]=\frac{8+|x y|}{16}\left[|x|+|y|+\frac{|x|^{2}}{10}+\frac{|y|^{2}}{12}\right] \\
& \quad=\frac{1}{16}\left[\left(8(|x|+|y|)+\frac{|x|^{2}}{10}+\frac{|y|^{2}}{12}\right)\right. \\
&\left.+|x y|\left((|x|+|y|)+\frac{|x|^{2}}{10}+\frac{|y|^{2}}{12}\right)\right] \\
&+\frac{7}{16}\left(d_{k}(x, y)\right) . \\
& \quad \frac{1}{16}\left[\left(8(|x|+|y|)+\frac{|x|^{2}}{10}+\frac{|y|^{2}}{12}\right)\right. \\
&\left.+\left((|x|+|y|)+\frac{|x|^{2}}{10}+\frac{|y|^{2}}{12}\right)\right] \\
&+\frac{1}{16}\left(9(|x|+|y|)+\frac{|x|^{2}}{5}+\frac{|y|^{2}}{6}\right) \leq \frac{9}{16}(|x| \\
&+
\end{aligned}
$$

Hence, $T$ has a deqb- weak contraction property of Theorem 20 and $x=0$ is the unique fixed point of $T$. 
Theorem 22. Let $\left(X, d_{k}\right)$ be a complete dislocated quasi extended b-metric space, $G$ and $H$ be closed subsets of $X$ and let $0<\lambda<1$. If $T: G \cup H \longrightarrow G \cup H$ is a cyclic, continuous function which satisfy the conditions

$$
k(T x, T y) d_{k}(T x, T y) \leq \lambda \varphi\left(d_{k}(x, y)\right),
$$

where $\varphi:[0, \infty) \longrightarrow[0, \infty)$ be a $\varphi$ nondecreasing and continuous function, $\varphi(t)=0$ if only if $t=0$ and $\varphi(\lambda t) \leq$ $\lambda \varphi(t), \varphi^{n+1}(t) \leq \varphi^{n}(t), \varphi^{n+1}(t)=\varphi\left(\varphi^{n}(t)\right)$, for $n=1,2,3, \ldots$, and $\lim _{n, m \rightarrow \infty} k\left(x_{n}, x_{m}\right)<1 / \lambda$.

Then $T$ has unique fixed point in $G \cap H$.

Proof. Since $T$ is a cyclic map, for $x_{0} \in G$, then $T x_{0} \in H$ and $T^{2} x_{0} \in G$. Define a sequence $\left\{x_{n}\right\}$, where $x_{n}=T x_{n-1}=T^{n} x_{0}$. So we have $x_{2 n} \in G$ and $x_{2 n-1} \in H$ for $n=1,2,3 \ldots$

Since $k(x, y) \geq 1$ and $0<\lambda<1$ then for all $n \in N$, we have

$$
\begin{aligned}
k & \left(x_{n}, x_{n+1}\right) d_{k}\left(x_{n}, x_{n+1}\right) \\
& =k\left(T x_{n-1}, T x_{n}\right) d_{k}\left(T x_{n-1}, T x_{n}\right) \\
& \leq \lambda \varphi\left(d_{k}\left(x_{n-1}, x_{n}\right)\right) \leq \lambda \varphi\left(\lambda \varphi\left(d_{k}\left(x_{n-2}, x_{n-1}\right)\right)\right) \\
& =\lambda^{2} \varphi^{2}\left(\left(d_{k}\left(x_{n-2}, x_{n-1}\right)\right)\right) \leq \lambda^{n} \varphi^{n}\left(\left(d_{k}\left(x_{0}, x_{1}\right)\right)\right) .
\end{aligned}
$$

We have

$$
d_{k}\left(x_{n}, x_{n+1}\right) \leq k\left(x_{n}, x_{n+1}\right) d_{k}\left(x_{n}, x_{n+1}\right) \leq \lambda^{n} \varphi^{n}\left(t_{0}\right),
$$

where $t_{0}=d_{k}\left(x_{0}, x_{1}\right)$.

By using (2) and (52), we have

$$
\begin{aligned}
d_{k} & \left(x_{n}, x_{m}\right) \leq k\left(x_{n}, x_{m}\right)\left(d_{k}\left(x_{n}, x_{n+1}\right)\right. \\
& \left.+d_{k}\left(x_{n+1}, x_{m}\right)\right) \leq k\left(x_{n}, x_{m}\right) \\
& \left.\cdot\left(d_{k}\left(x_{n}, x_{n+1}\right)+d_{k}\left(x_{n+1}, x_{m}\right)\right)\right) \leq k\left(x_{n}, x_{m}\right) \\
& \cdot\left(\lambda^{n} \varphi^{n}\left(t_{0}\right)+d_{k}\left(x_{n+1}, x_{m}\right)\right) \leq k\left(x_{n}, x_{m}\right)\left(\lambda^{n}\right. \\
& \cdot \varphi^{n}\left(t_{0}\right)+k\left(x_{n+1}, x_{m}\right)\left(d_{k}\left(x_{n+1}, x_{n+2}\right)\right. \\
& \left.+d_{k}\left(x_{n+2}, x_{m}\right)\right) \leq k\left(x_{n}, x_{m}\right)\left(\lambda^{n} \varphi^{n}\left(t_{0}\right)\right. \\
& \left.+k\left(x_{n+1}, x_{m}\right) d_{k}\left(x_{n+1}, x_{n+2}\right)+d_{k}\left(x_{n+2}, x_{m}\right)\right) \\
& \leq k\left(x_{n}, x_{m}\right)\left(\lambda^{n} \varphi^{n}\left(t_{0}\right)+k\left(x_{n+1}, x_{m}\right)\right. \\
& \cdot\left(\lambda^{n+1} \varphi^{n+1}\left(t_{0}\right)+d_{k}\left(x_{n+2}, x_{m}\right)\right) \leq k\left(x_{n}, x_{m}\right) \\
& \cdot\left(\lambda^{n} \varphi^{n}\left(t_{0}\right)+k\left(x_{n+1}, x_{m}\right) \lambda^{n+1} \varphi^{n+1}\left(t_{0}\right)\right. \\
& \left.+k\left(x_{n+1}, x_{m}\right) d_{k}\left(x_{n+2}, x_{m}\right)\right) \leq k\left(x_{n}, x_{m}\right)\left(\lambda^{n}\right. \\
& +\varphi^{n}\left(t_{0}\right)+k\left(x_{n+1}, x_{m}\right) \lambda^{n+1} \varphi^{n+1}\left(t_{0}\right) \\
& +k\left(x_{n+1}, x_{m}\right) k\left(x_{n+2}, x_{m}\right)\left(d_{k}\left(x_{n+2}, x_{n+3}\right)\right. \\
+ & \left.d_{k}\left(x_{n+3}, x_{m}\right)\right) \leq k\left(x_{n}, x_{m}\right)\left(\lambda^{n} \varphi^{n}\left(t_{0}\right)\right. \\
+ & k\left(x_{n+1}, x_{m}\right) \lambda^{n+1} \varphi^{n+1}\left(t_{0}\right)+k\left(x_{n+1}, x_{m}\right)
\end{aligned}
$$

$$
\begin{aligned}
& \cdot k\left(x_{n+2}, x_{m}\right)\left(\lambda^{n+2} \varphi^{n+2}\left(t_{0}\right)+d_{k}\left(x_{n+3}, x_{m}\right)\right) \\
& \leq k\left(x_{n}, x_{m}\right)\left(\lambda^{n} \varphi^{n}\left(t_{0}\right)+k\left(x_{n+1}, x_{m}\right)\right. \\
& \cdot \lambda^{n+1} \varphi^{n+1}\left(t_{0}\right)+k\left(x_{n+1}, x_{m}\right) k\left(x_{n+2}, x_{m}\right) \\
& \cdot \lambda^{n+1} \varphi^{n+2}\left(t_{0}\right)+\cdots+k\left(x_{n+1}, x_{m}\right) k\left(x_{n+2}, x_{m}\right) \\
& \cdots \cdot k\left(x_{m-1}, x_{m}\right) \lambda^{m-1} \varphi^{m-1}\left(t_{0}\right) \\
& =\sum_{i=0}^{m-n-1} \lambda^{n+i} \varphi^{n+i}\left(t_{0}\right) \prod_{j=0}^{i} k\left(x_{n+j}, x_{m}\right) \\
& =\sum_{i=n}^{m-1} \lambda^{i} \varphi^{i}\left(t_{0}\right) \prod_{j=0}^{i} k\left(x_{n+j}, x_{m}\right) .
\end{aligned}
$$

We have

$$
d_{k}\left(x_{n}, x_{m}\right) \leq \sum_{i=n}^{m-1} \lambda^{i} \varphi^{i}\left(t_{0}\right) \prod_{j=0}^{i} k\left(x_{n+j}, x_{m}\right) .
$$

Let $a_{i}=\lambda^{i} \varphi^{i}\left(t_{0}\right) \prod_{j=0}^{i} k\left(x_{n+j}, x_{m}\right)$.

Since $\varphi^{n+1}(t) \leq \varphi^{n}(t)$ we have

$$
\begin{aligned}
\frac{a_{i+1}}{a_{i}} & =\frac{\varphi^{n+i+1}\left(t_{0}\right)}{\varphi^{n+i}\left(t_{0}\right)} \lambda k\left(x_{n+i+1}, x_{m}\right) \\
& \leq \lambda k\left(x_{n+i+1}, x_{m}\right) . \\
\lim _{i \rightarrow \infty} \frac{a_{i+1}}{a_{i}} & =\lim _{i \rightarrow \infty} \frac{\varphi^{n+i+1}\left(t_{0}\right)}{\varphi^{n+i}\left(t_{0}\right)} \lambda k\left(x_{n+i+1}, x_{m}\right) \\
& \leq \lim _{i \rightarrow \infty} \lambda k\left(x_{n+i+1}, x_{m}\right)<1 .
\end{aligned}
$$

By using the ratio test criteria, we get $\sum_{i=0}^{\infty} \lambda^{n+i} \varphi^{n+i}\left(t_{0}\right) \prod_{j=0}^{i} k\left(x_{n+j}, x_{m}\right)=\sum_{i=0}^{\infty} a_{i}$ convergence.

Let $S_{p}=\sum_{i=0}^{p} a_{i}$, then from (54), we get

$$
\begin{aligned}
d_{k}\left(x_{n}, x_{m}\right) & \leq \sum_{i=0}^{m-n-1} \lambda^{n+i} \varphi^{n+i}\left(t_{0}\right) \prod_{j=0}^{i} k\left(x_{n+j}, x_{m}\right) \\
& =\sum_{i=n}^{m-1} a_{i}=S_{m-1}-S_{n-1} \leq\left|S_{m-1}-S_{n-1}\right| .
\end{aligned}
$$

Thus for $n, m \longrightarrow \infty$ we get $d_{k}\left(x_{n}, x_{m}\right) \longrightarrow 0$. Hence $\left\{x_{n}\right\}$ is a Cauchy sequence in $X$.

Since $X$ complete, there exists $x^{*} \in X$ such that $d_{k}\left(x_{n}, x^{*}\right) \longrightarrow 0$ for $n \longrightarrow \infty$.

Similarly, we can have $d_{k}\left(x^{*}, x_{n}\right) \longrightarrow 0$.

Since the sequence $\left\{x_{2 n}\right\} \in G,\left\{x_{2 n-1}\right\} \in H$ and $G, H$ closed, thus we have $x^{*} \in G \cap H$. 
Now we prove that $x^{*}$ is a fixed point of T. Using (2) and (11) we have

$$
\begin{aligned}
& d_{k}\left(T x^{*}, x^{*}\right) \leq k\left(T x^{*}, x^{*}\right) \\
& \cdot\left(d\left(T x^{*}, T x_{n-1}\right)+d\left(T x_{n-1}, x^{*}\right)\right) \leq k\left(T x^{*}, x^{*}\right) \\
& \cdot\left(k\left(T x^{*}, T x_{n-1}\right) d\left(T x^{*}, T x_{n-1}\right)+d\left(T x_{n-1}, x^{*}\right)\right) \\
& \leq k\left(T x^{*}, x^{*}\right)\left(\lambda \varphi\left(d\left(x^{*}, x_{n-1}\right)\right)+d\left(x_{n}, x^{*}\right)\right) .
\end{aligned}
$$

Using continuity of $\varphi$, and $\varphi(0)=0$, then for $n \longrightarrow \infty$, we have $d_{k}\left(T x^{*}, x^{*}\right) \leq \lambda k\left(T x^{*}, x^{*}\right) \varphi(0) \leq 0$.

Thus $d_{k}\left(T x^{*}, x^{*}\right)=0$, hence $T x^{*}=x^{*}$.

Now we have to show that $T$ has unique fixed point in $X$. Suppose $u$ is an another fixed point of $T$,

$$
\begin{aligned}
d_{k}\left(x^{*}, u\right) & =d_{k}\left(T x^{*}, T u\right) \\
& \leq k\left(T x^{*}, T u\right) d_{k}\left(T x^{*}, T u\right) \\
& \leq \lambda \varphi\left(d_{k}\left(x^{*}, u\right)\right) .
\end{aligned}
$$

We have

$$
(1-\lambda) \varphi d_{k}\left(x^{*}, u\right) \leq 0 .
$$

Since $1-\lambda>0$ thus we get $\varphi\left(d_{k}\left(x^{*}, u\right)\right) \leq 0$. Since $\varphi \geq 0$, then $\varphi\left(d_{k}\left(x^{*}, u\right)\right)=0$. Which implies that $d_{k}\left(x^{*}, u\right)=0$, so we have $x^{*}=u$.

Example 23. Let $X=[-1,1]$ and $\left(X, d_{k}\right)$ be a dislocated quasi extended b-metric space which in Example 8. Let $T: G \cup$ $H \longrightarrow G \cup H$ be a function defined by $T x=-x^{3} / 8$, where $G=$ $[-1,0], H=[0,1]$. Let $\varphi:[0, \infty) \longrightarrow[0, \infty)$ be a function and defined as, $\varphi(t)=(3 / 4) t^{2}$ and $\lambda=1 / 4$.

In fact, it clear that $\varphi(\lambda t) \leq \lambda \varphi(t), \varphi^{n+1}(t) \leq \varphi^{n}(t)$ and $T$ is cyclic, since $T(G) \subseteq H$ and $T(H) \subseteq G$.

Since $x_{n}, x_{m} \in X=[-1,1]$ and $k\left(x_{n}, x_{m}\right)=\left(2+x_{n} x_{m}\right) / 2$, it is easy to show that $\lim _{n, m \rightarrow \infty} k\left(x_{n}, x_{m}\right)<1 / \lambda$.

Now, we have to show that

$$
\begin{aligned}
& k(T x, T y) d_{k}(T x, T y) \leq \lambda \varphi\left(d_{k}(x, y)\right) . \\
& k(T x, T y) d_{k}(T x, T y) \\
& \quad=k\left(\frac{-x^{3}}{8}, \frac{-y^{3}}{8}\right) d_{k}\left(\frac{-x^{3}}{8}, \frac{-y^{3}}{8}\right) \\
& \quad=\frac{2+\left|\left(-x^{3} / 8\right)\left(-y^{3} / 8\right)\right|}{2} d_{k}\left(\frac{-x^{3}}{8}, \frac{-y^{3}}{8}\right) \\
& \quad \leq \frac{2+\left|x^{3} y^{3}\right|}{2} d_{k}\left(\frac{-x^{3}}{8}, \frac{-y^{3}}{8}\right) \\
& \quad \leq \frac{2+\left|x^{3} y^{3}\right|}{2} \mid\left(\left|\frac{-x^{3}}{8}\right|+\left|\frac{-y^{3}}{8}\right|\right)+\frac{\left|-x^{3} / 8\right|^{2}}{5}
\end{aligned}
$$

$$
\begin{aligned}
& \left.+\frac{\left|-y^{3} / 8\right|^{2}}{6}\right]=\frac{2+\left|x^{3} y^{3}\right|}{16}\left[\left|x^{3}\right|+\left|y^{3}\right|+\frac{\left|x^{3}\right|^{2}}{40}\right. \\
& \left.+\frac{\left|y^{3}\right|^{2}}{48}\right]=\frac{3}{16}\left[\left|x^{3}\right|+\left|y^{3}\right|+\frac{\left|x^{3}\right|^{2}}{40}+\frac{\left|y^{3}\right|^{2}}{48}\right] \\
& \leq \frac{3}{16}\left(\left|x^{2}\right|+\left|y^{2}\right|+\frac{\left|x^{2}\right|^{2}}{25}+\frac{\left|y^{2}\right|^{2}}{36}\right) \leq \frac{3}{16}((|x| \\
& \left.+|y|+\frac{|x|^{2}}{5}+\frac{|y|^{2}}{6}\right)^{2}=\frac{1}{4} \varphi\left(d_{k}(x, y)\right) .
\end{aligned}
$$

Hence, $T$ has a deqb-weak contraction property of Theorem 22 and $x=0$ is the unique fixed point of $T$.

\section{Conclusion}

In this article, we considered and proved the fixed point theorems for cyclic weakly contraction mapping in complete dislocated quasi extended $b$-metric space. These results generalize the recent results of Samreen [14] and Rahman [9], which was in our results more general in the sense by utilizing dislocated quasi extended $b$-metric and cyclic weakly contraction. Furthermore, In Theorems 16, 18, 20, and 22 one can derive several consequences in dislocated quasi $b$ metric by letting $k(x, y)=K \geq 1$ and in dislocated quasi metric by letting $k(x, y)=1$.

\section{Data Availability}

No data were used to support this study.

\section{Conflicts of Interest}

The author declares that there are no conflicts of interest.

\section{Acknowledgments}

The author is grateful to Hasanuddin University for financial support of this work by BMIS Research Project 2017 no. 3556/UN.4.3.2/LK.23/2017.

\section{References}

[1] I. A. Bakhtin, "The contraction mapping principle in almost metric space," Functional Analysis and its Applications, vol. 30, pp. 26-37, 1989.

[2] G. S. Saluja, "Some unique fixed point theorems for rational expressions in cone $b$-metric spaces," Nonlinear Analysis Forum, vol. 21, no. 2, pp. 183-193, 2016.

[3] Z. Mostefaoui, M. Bousselsal, and J. K. Kim, "Some results in fixed point theory concerning rectangular b-metric spaces," Nonlinear Functional Analysis and Applications, vol. 24, no. 1, pp. 49-59, 2019. 
[4] B. S. Chaudhury, P. N. Dutta, and P. Maity, "Weak contraction principle in b-metric spaces," Journal of Mathematics and Informatics, vol. 6, pp. 15-19, 2016.

[5] A. H. Ansari, S. Chandok, and C. Ionescu, "Fixed point theorems on $b$-metric spaces for weak contractions with auxiliary functions," Journal of Inequalities and Applications, vol. 2014, article no. 429, 2014.

[6] M. H. Shah and N. Hussain, "Nonlinear contractions in partially ordered quasi $b$-metric spaces," Communications of the Korean Mathematical Society, vol. 27, no. 1, pp. 117-128, 2012.

[7] C. Zhu, C. Chen, and X. Zhang, "Some results in quasi-bmetric-like spaces," Journal of Inequalities and Applications, vol. 2014, no. 1, p. 437, 2014.

[8] M. Cvetković, E. Karapınar, and V. Rakocević, "Some fixed point results on quasi-b-metric-like spaces," Journal of Inequalities and Applications, vol. 1, no. 374, 2015.

[9] N. Hussain, J. R. Roshan, V. Parvaneh, and M. Abbas, "Common fixed point results for weak contractive mappings in ordered $b$ dislocated metric spaces with applications," Journal of Inequalities and Applications, vol. 2013, article no. 486, 2013.

[10] T. Rasham, A. Shoaib, N. Hussain, B. AS Alamri, and B. M. Arshad, "Multivalued fixed point results in dislocated b-metric spaces with application to the system of nonlinear integral equations," Symmetry, vol. 11, no. 1, article no. 40, 2019.

[11] M. U. Rahman and M. Sarwar, "Dislocated quasi b-metric space and fixed point theorems," Electronic Journal of Mathematical Analysis and Applications, vol. 4, no. 2, pp. 16-24, 2016.

[12] C. Klin-eam and C. Suanoom, "Dislocated quasi-b-metric spaces and fixed point theorems for cyclic contractions," Fixed Point Theory and Applications, vol. 2015, article no. 74, 12 pages, 2015.

[13] C. Suanoom, C. Klin-eam, and S. Suantai, "Dislocated quasib-metric spaces and fixed point theorems for cyclic weakly contractions," Journal of Nonlinear Sciences and Applications, vol. 09, no. 05, pp. 2779-2788, 2016.

[14] S. K. Tiwari and V. Vishwakarma, "Dislocated quasi b-metric Space and new common fixed point results," International Journal for Science and Advance Research, vol. 3, no. 7, pp. 60-64, 2017.

[15] T. Kamran, M. Samreen, and Q. U. L. Ain, "A generalization of b-Metric space and some fixed point theorems," Mathematics, vol. 5, no. 2, article no. 19, 2017.

[16] M. Samreen, T. Kamran, and M. Postolache, "Extended bmetric space, extended $b$-comparison function and nonlinear contractions," University of Bucharest Scientific Bulletin Series A Applied Mathematics and Physics, vol. 4, pp. 21-28, 2018.

[17] B. Alqahtani, A. Fulga, and E. Karapınar, "Common fixed point results on an extended b-metric space," Journal of Inequalities and Applications, vol. 1, no. 158, 2018.

[18] B. Alqahtani, E. Karapınar, and A. Öztürk, "On $(\alpha, \psi)-\mathrm{k}-$ contractions in the extended b-metric space," Filomat, vol. 32, no. 15, pp. 5337-5345, 2018.

[19] B. E. Rhoades, "Some theorems on weakly contractive maps," Nonlinear Analysis-theory Methods \& Applications, vol. 47, no. 4, pp. 2683-2693, 2001.

[20] K. Zoto, E. Hoxha, and A. Isufati, "Fixed point theorems for cyclic contractions in dislocated metric spaces," Proceedings in ARSA-Advanced Research in Scientific Areas, vol. 4, 2013. 


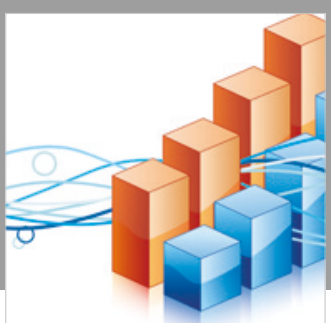

Advances in

Operations Research

\section{-n-m}
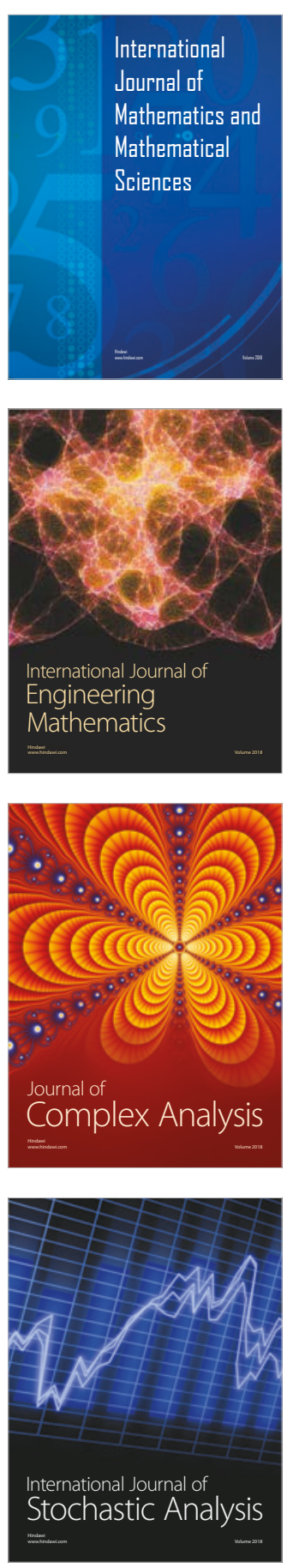
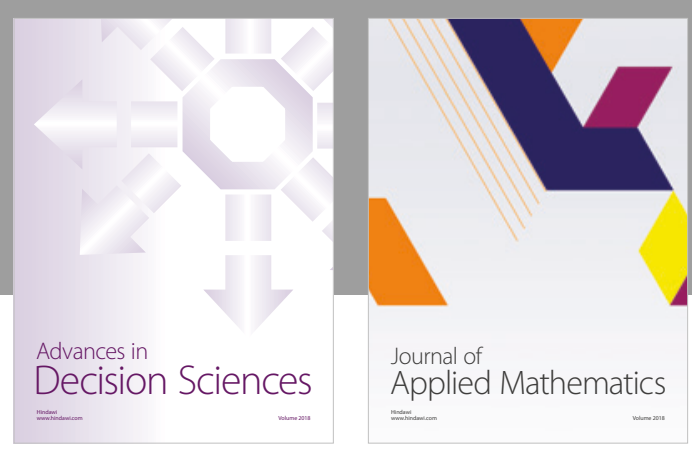

Journal of

Applied Mathematics
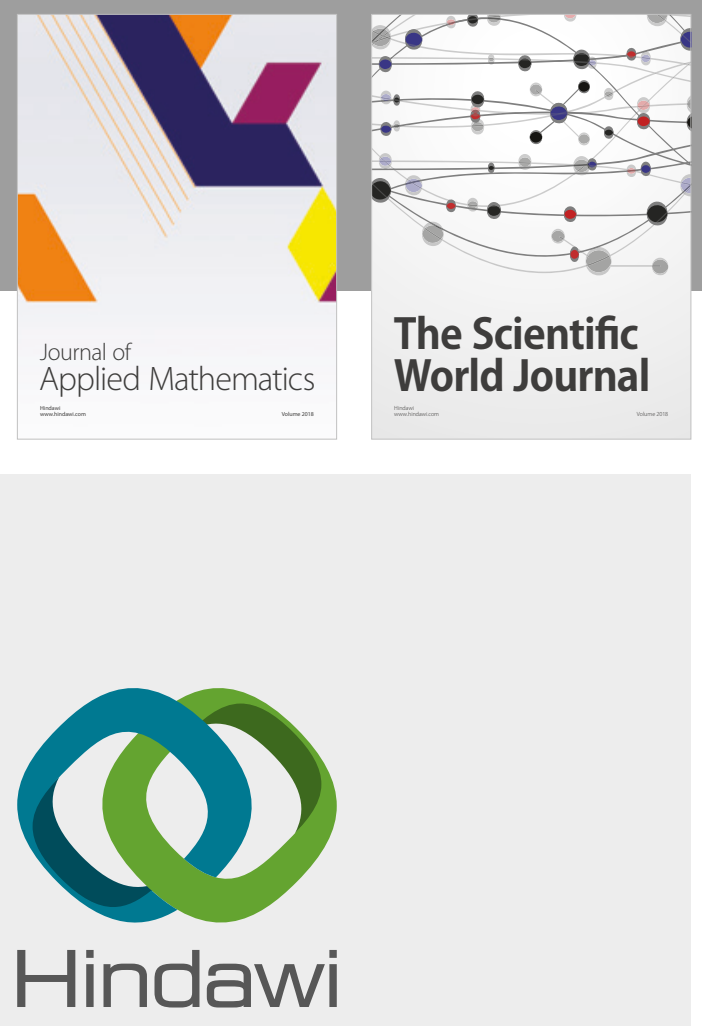

Submit your manuscripts at

www.hindawi.com

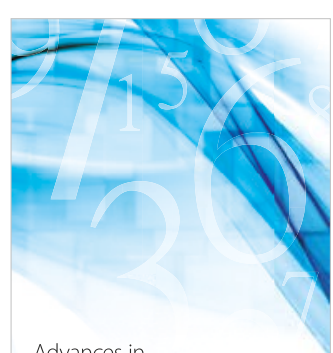

Advances in
Numerical Analysis
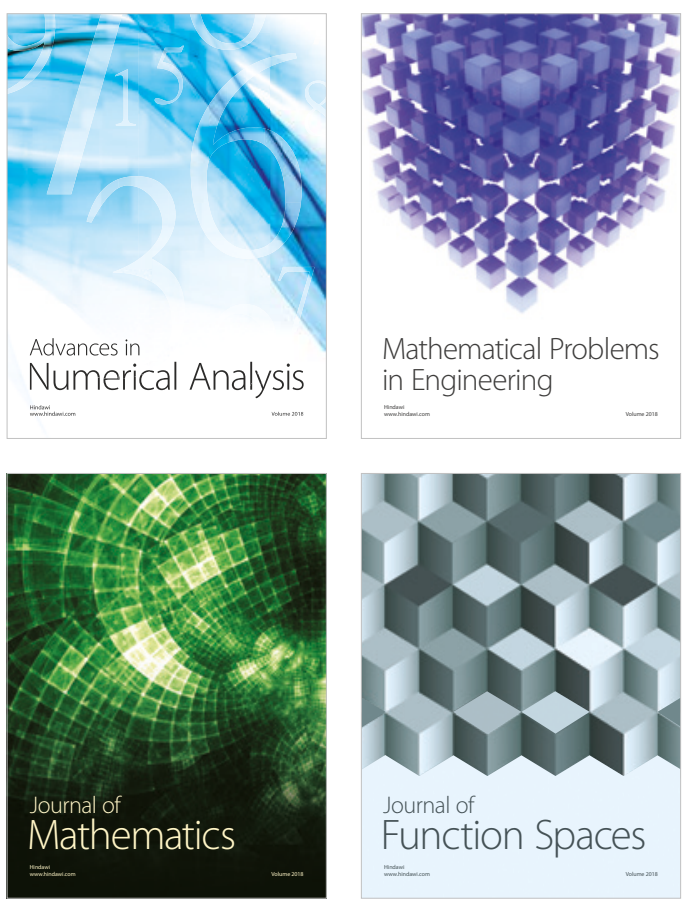

Mathematical Problems in Engineering

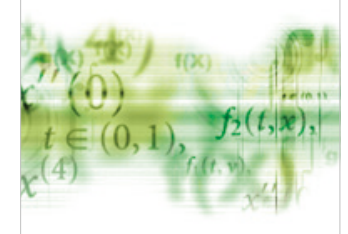

International Journal of

Differential Equations

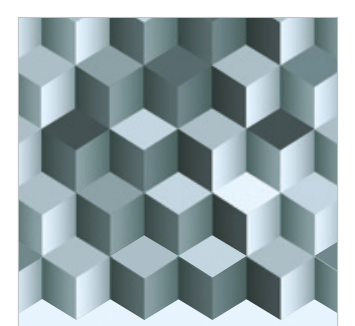

Journal of

Function Spaces

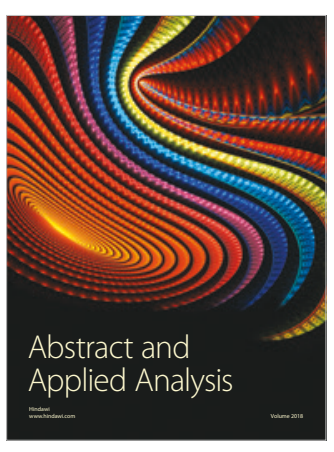

The Scientific

World Journal

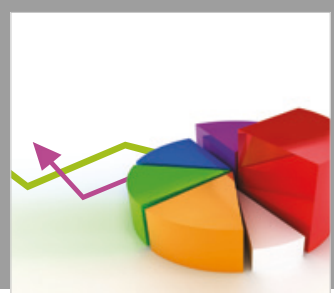

Journal of

Probability and Statistics
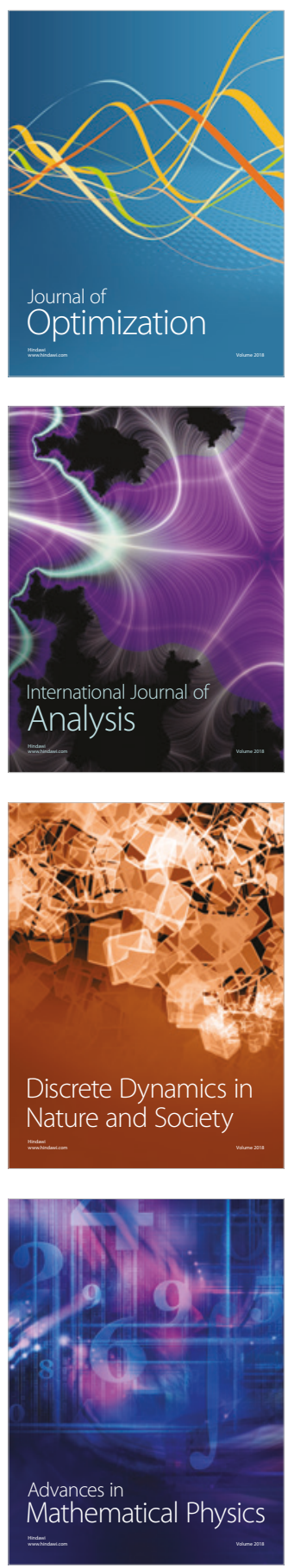